\title{
CD151 in cancer progression and metastasis: a complex scenario
}

Rafal Sadej ${ }^{1,3}$, Alicja Grudowska', Lukasz Turczyk ${ }^{1}$, Radzislaw Kordek² and Hanna M Romanska ${ }^{2,3}$

Originally identified as a molecular organizer of interacting proteins into tetraspanin-enriched microdomains, the tetraspanin CD151 has now been shown to be involved in tumour progression. Increasing evidence emerging from in vitro, in vivo and clinical analyses implicates this tetraspanin in supporting growth of various types of tumours at different levels. It affects both cell autonomous behavior and communication with neighboring cells and the microenvironment. CD151 regulates post-adhesion events, that is, cell spreading, migration and invasion including subsequent intravasation and formation of metastasis. Present on both neoplastic and endothelial cells, CD151 is engaged in promotion of tumour neovascularization. The molecular mechanism of CD151 in cancer is based on its ability to organize distribution and function of interacting proteins, ie, laminin-binding integrins $(\alpha 3 \beta 1, \alpha 6 \beta 1$ and $\alpha 6 \beta 4)$, receptors for growth factors (HGFR, EGFR and TGF- $\beta 1$ R) and matrix metalloproteinases (MMP-7, MMP-2 and MMP-9), which indicates its importance in disease development. Results of clinical analyses of CD151 expression in different types of cancer and a large number of in vivo models demonstrate its impact on tumour growth and invasion and implicate CD151 as a valuable diagnostic and prognostic marker as well as a potential target for anti-cancer therapy. Laboratory Investigation (2014) 94, 41-51; doi:10.1038/labinvest.2013.136; published online 18 November 2013

KEYWORDS: cancer progression; CD151; laminin-binding integrins; metastasis; tetraspanins

\section{INTRODUCTION}

Tumour cells progress through multiple orchestrated steps before metastatic lesions are established in distant organs. These steps include detachment from the primary tumour mass, invasion of the basement membrane, migration through the surrounding extracellular matrix (ECM) followed by intravasation and extravasation and, finally, colonization of a secondary anatomical site. Successful completion of this sequence requires intrinsic changes enabling phenotypic transformation of the cell and, at every step, its coordinated communication with the tumour's microenvironment. A complex network of intricate tumourstroma interactions involves integrin-dependent adhesion and cell migration on the ECM, ECM degradation by matrix metalloproteinases (MMPs), and activation of signalling pathways responsible for cell survival, proliferation and motility, triggered by growth factor receptors stimulated by the environment.
It is well recognized that acquisition of an invasive phenotype by the cell requires activation of a molecular programme mediated by complex signalling networks. The mechanism of this transition is not fully understood but a number of regulators have been implicated. One of these is CD151 (SFA-1, PETA3), a member of the evolutionarily conserved transmembrane- 4 family (tetraspanins), expressed in almost all cell types and tissues. ${ }^{1,2}$ Tetraspanins are involved in regulation of a variety of both normal physiological (eg, cell adhesion, motility, activation and proliferation) and pathological processes (eg, tumour progression and metastasis). ${ }^{3,4}$ Increasing clinical evidence seems to points to their potential for cancer prognostication. ${ }^{5}$ CD151 was the first tetraspanin associated with cancer development. Its role in promotion of invasion and migration has been demonstrated in numerous in vitro and in vivo models. ${ }^{6-10}$

CD151 was found to participate in nearly all stages of cancer progression. Its involvement in the early events of

\footnotetext{
${ }^{1}$ Department of Molecular Enzymology, Intercollegiate Faculty of Biotechnology, University of Gdansk and Medical University of Gdansk, Gdansk, Poland and ${ }^{2}$ Department of Pathology, Medical University of Łódź, Łódź, Poland

Correspondence: Dr R Sadej, Department of Molecular Enzymology, Intercollegiate Faculty of Biotechnology, University of Gdansk and Medical University of Gdansk, Gdansk 80-210, Poland or Dr HM Romanska, Department of Pathology, Medical University of Łódź, Łódź 90-410, Poland.

E-mail: rsadej@gumed.edu.pl or hanna.romanska@gmail.com

${ }^{3}$ These authors contributed equally to this work.

Received 8 July 2013; accepted 22 October 2013
} 
tumour development was demonstrated in diverse biological contexts. For example, CD151 supported de novo carcinogenesis in a chemically induced mouse model of squamous cell carcinoma (SCC) manifested in increased incidence, multiplicity, tumour size and progression to a malignant form of skin SCC. ${ }^{11}$ In breast cancer (BCa), CD151 had a critical role in controlling proliferation of cells in ductal carcinoma in situ (DCIS), a pre-invasive form of BCa. ${ }^{12}$ Its contribution to further steps of cancer development includes maintenance of tumour neovascularization, ${ }^{13-15}$ regulation of cell polarity, motility and invasion. ${ }^{16-19}$ For example, treatment of cells with anti-CD151 mAb impaired not only the epithelial polarization but also the assembly of cortical actin belts, revealing a significant contribution of this tetraspanin to cytoskeleton dynamics. ${ }^{20}$ The molecular mechanism of CD151-coordinated actin contraction may involve tetraspanin's ability to control the function of small GTPases. ${ }^{20-22}$ CD151 has also been implicated in epithelialmesenchymal transition (EMT) of hepatocellular carcinoma (HCC) promoting a motile phenotype of these cells. ${ }^{23}$ CD151, together with integrin $\alpha 3 \beta 1$, located at the periphery of specialized surface protrusions-invadopodia, was found to regulate expression and activity of MMPs. ${ }^{7,24}$

Finally, numerous studies using in vitro and in vivo models of cancer implicate CD151 in the promotion of metastasis. For example, increased CD151 expression appeared to be critical at the early steps of metastatic lesion formation by an epidermoid carcinoma cell line. ${ }^{6}$ CD151 facilitated the metastatic process by enhancing cell migration and intravasation. ${ }^{25}$ It was shown that CD151 took part in a retention of cancer cells in the lung vascular bed and formation of lung metastasis by fibrosarcoma, breast and colon cancer cell lines. ${ }^{8,26}$ Moreover, deletion of the tetraspanin had no impact on cancer initiation in CD151 knock-out mouse crossed with transgenic adenocarcinoma of mouse prostate (TRAMP) model, but its ablation significantly impaired formation of pulmonary metastases. ${ }^{27}$

A series of excellent reviews discuss a contribution of various tetraspanins to carcinogenesis. ${ }^{2,5,28}$ Here, we provide the first updated summary of existing literature focussing specifically on CD151 and its role in cancer progression.

\section{CD151 STRUCTURE AND FUNCTION}

CD151 gene is located on chromosome 11p15.5. ${ }^{29}$ It was first cloned as PETA-3 from a megakaryoblastic leukaemia cell line and its cDNA, with an open reading frame comprising 253 amino acids, encodes a protein of the molecular mass of $28 \mathrm{kDa} .{ }^{30}$ Tetraspanin CD151 consists of four transmembrane domains, two extracellular (EC1 and EC2) and one intracellular loop, and $\mathrm{NH} 2-$ and $\mathrm{COOH}$-terminal cytoplasmic domains. $^{31}$

CD151 is clustered at the cell membrane in specialized multimeric aggregates, ie, tetraspanin-enriched microdomains (TEMs or 'tetraspanin web'). It is postulated that at the molecular level, this tetraspanin is a lateral organizer and modulator of activities of transmembrane proteins such as adhesion molecules, ie, laminin-binding integrins, ${ }^{19,32}$ growth factor receptors (HGFR, EGFR and TGF $\beta$ R) 26,33,34 $^{26}$ and proteases. ${ }^{24,35}$ The functional association with numerous proteins indispensable for tumour progression implies a regulatory role for CD151 at different stages of disease development.

\section{Master Regulator of Laminin-Binding Integrins}

CD151 forms stable, lateral complexes with laminin-binding integrins, ie, $\alpha 3 \beta 1, \alpha 6 \beta 1$ and $\alpha 6 \beta 4$ crucial in cancer cell migration and invasion ${ }^{36-38}$ (Figure 1). Integrin subunits $\alpha 3$ and $\alpha 6$ directly interact with CD151 through the QRD ${ }^{194-196}$ site at the extracellular loop of the protein. Mutation of this sequence to $\mathrm{INF}^{194-196}$ abolished formation of the complex, ${ }^{32}$ which impeded many integrin-dependent cell functions. However, it has been recently suggested that this region is not an essential component but rather a strengthening factor of the CD151-integrin complex. ${ }^{39}$

In cells expressing both CD151 and laminin-binding integrins, CD151 is thought to be principally involved in all types of integrin-mediated cellular behavior through a variety of mechanisms such as regulation of integrin-ligand interaction and integrin-triggered signalling, direction of integrin intracellular trafficking, recycling, and their compartmentalization on the cell surface. ${ }^{3}$

\section{TUMOR MICROENVIRONMENT}

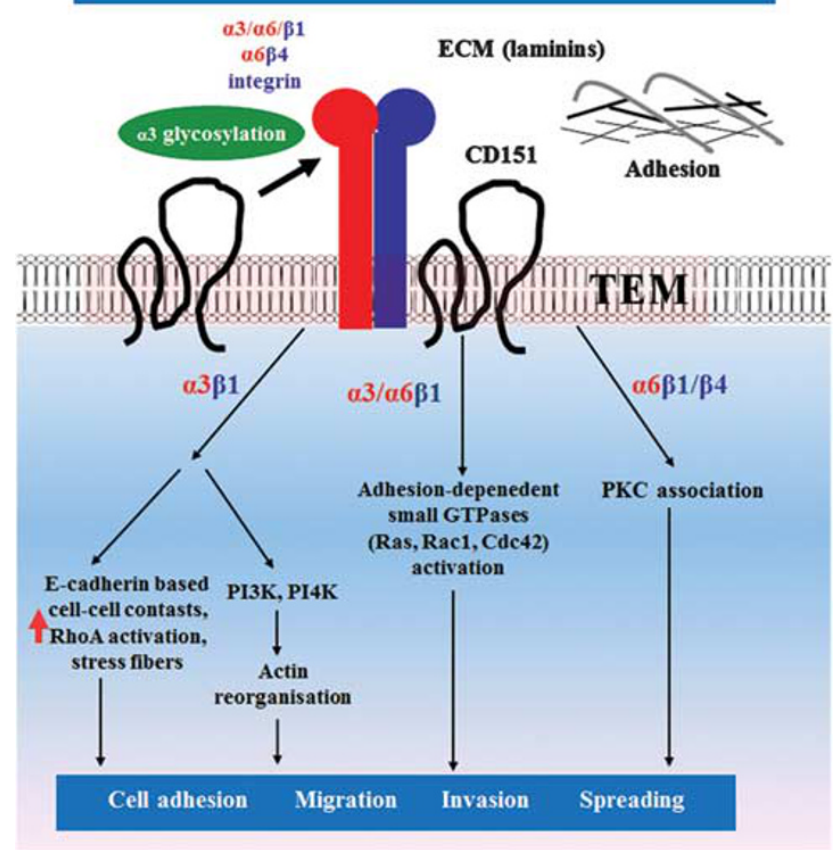

Figure 1 Complexity of CD151 action in cancer progression-regulation of integrin-dependent events. CD151 forms direct and stable complexes with laminin-binding integrins and regulates their activities, which results in modulation of adhesion, spreading, migration, invasion and metastasis. 


\section{Modulation of Integrin-Ligand interactions}

Modulation of integrin-ligand interactions, in particular, has an impact on cellular responses such as cell attachment, spreading and migration. In A549 human lung adenocarcinoma cells, CD151-free pool of integrin $\alpha 3 \beta 1$ showed markedly impaired ability to interact with its highest affinity ligand, laminin-511/521. Nishiuchi et al ${ }^{40}$ concluded that an association with CD151 regulates the conformation of $\alpha 3 \beta 1$ integrin, which sustains its activated state. However, the authors used $\alpha 3 \beta 1$-containing liposomes (with or without $\mathrm{CD} 151)$ and tested their adhesion to laminin-511/521 or AG89 mAb (selectively recognizing activated $\beta 1$-containing integrins). It is therefore highly possible that CD151 selfassociated and formed $\alpha 3 \beta 1$-enriched domains in liposomes increasing not affinity but simply its avidity to laminin 551/ 521. Furthermore, as the antibody AG89 was immobilized on plastic surface, the assay measured adhesion of liposomes rather than antibody binding. Several other reports seem to be in agreement that CD151 has no effect on integrin conformation/activation. ${ }^{34,41,42}$ As demonstrated by Yamada and co-workers in A549 cells, a CD151 was involved in modulation of two independent aspects of integrin $\alpha 3 \beta 1$ functions, namely, potentiation of $\alpha 3 \beta 1$-mediated cell adhesion and promotion of $\alpha 3 \beta 1$-stimulated signalling events involving tyrosine phosphorylation. Knockdown of CD151 resulted in formation of aberrant membrane protrusions and reduction in phosphorylation of focal adhesion kinase (FAK), Src, p130Cas and paxillin in cells grown on laminin-511. ${ }^{43}$

\section{Post-Translational Modifications and Trafficking}

CD151 has the ability to regulate post-translational modifications and trafficking of associated partners. CD151 interacting with integrins $(\alpha 3 \beta 1$ and $\alpha 6 \beta 1)$ undergoes endocytosis and accumulates in intracellular vesicular compartments. ${ }^{44}$ Thus by limiting the number of integrins on the cell surface, the protein can influence adhesive and migratory properties of carcinoma cells. ${ }^{45}$ CD151 contains an YRSL sequence, a tyrosine-based endocytosis/sorting motif, in its C-terminal cytoplasmic domain. Mutation of this region was proven to significantly attenuate internalization of the tetraspanin. Interestingly, disruption of CD151 trafficking completely abrogated CD151-promoted migration on laminin and diminished internalization of the associated integrins, indicating a critical role for integrin trafficking in regulation of cell motility. ${ }^{46}$ Therefore, the upregulation of CD151 frequently observed in malignancies may modify the rate of integrin trafficking resulting in facilitation of cell movement and promotion of invasiveness and metastatic potential. For example, in the pancreas, CD151 is expressed at low level in normal tissue, moderately expressed in chronic pancreatitis and highly expressed in pancreatic adenocarcinoma. The level of its expression is associated with those of CD151-interacting integrins- $\alpha 3 \beta 1$ and $\alpha 6 \beta 4$. Importantly, in cell lines derived from colorectal and pancreatic adenocarcinomas, CD151 was positively linked with cancer cell motility only when in complex with integrin $\alpha 6 \beta 4$. Transient internalization of $\alpha 6 \beta 4-C D 151$ complexes induced by treatment with a protein kinase $\mathrm{C}$ (PKC) activator, phorbol myristate acetate (PMA), was followed by a phenotypic change and an increase in cell motility. ${ }^{47}$

CD151 has been demonstrated to take part in the posttranslational modification of $\alpha 3$ integrin, ie, regulation of its glycosylation (not observed for other associated proteins, eg, $\alpha 6$ integrin or CD82). Changes in the glycosylation pattern of $\alpha 3 \beta 1$ integrin in CD151-depleted BCa cells correlated with a dramatic decrease in cell migration towards laminin-332. These findings suggest that modulation of the post-translational modifications of $\alpha 3 \beta 1$ integrin by CD151 might be one of the key mechanisms of the pro-migratory function of this tetraspanin. ${ }^{48}$

Site-directed mutagenesis has revealed that CD151 can be palmitoylated at multiple sites. This covalent modification was reported to be critical for the assembly and organization of the large network of TEMs and has a specific role in modulation of the activation state and signalling potential of laminin-binding integrins. ${ }^{49,50}$ Simultaneous mutation of six conserved cysteine residues (Cys11, Cys15, Cys79, Cys80, Cys242 and Cys243) in the large extracellular loop of CD151 eliminated palmitoylation and altered association with integrins, their partners and other tetraspanins (CD9 and CD63). Expression of the palmitoylation-deficient CD151 mutant did not change integrin-mediated cell spreading but increased the number of focal adhesions. In addition, the cells exhibited increased adhesion-induced phosphorylation of $\mathrm{PKB} / \mathrm{c}$-Akt, thus providing direct evidence for the role of CD151 in regulating the integrin-dependent phosphatidylinositol-3-kinase (PI3K) signalling pathway. It has also been shown that palmitoylation of CD151 has a pivotal role in the compartmentalization of laminin-binding integrins into TEMs and controls adhesion-dependent integrin signalling. ${ }^{50}$

\section{Compartmentalization on the Cell Surface}

Further mechanistic insight into the impact of CD151 on integrin function was provided by a study using the MDAMB-231 BCa cell line and single particle tracking. ${ }^{41}$ The authors demonstrated that CD151 influenced $\alpha 6$ integrin's diffusion mode, but not its magnitude at the cell membrane. CD151 restricted $\alpha 6$ integrin diffusion to random-confined (RCD) modes, typical for molecules able to explore larger areas of the cell membrane, suggesting that CD151 allows increased $\alpha 6$ integrin participation in cellular function. CD151 silencing resulted in the formation of a single or a limited number of adhesion sites, likely due to actindependent 'directed' motion (DMO) of $\alpha 6$ integrin. CD151 is thought to recruit PKC into closer proximity of $\alpha 6$ integrin. Deregulated DMO could not be reversed by phorbol ester, a treatment that activates and translocates PKC isozymes. As the absence of CD151 caused deregulation of $\alpha 6$-directed motion and signalling through disruption of conventional 
PKC isoform proximity to $\alpha 6$ integrin, it appears that $\alpha 6$ integrin can conduct outside-in signals and ECM cues to cellular events only when in complex with CD151.

CD151 regulates integrin subcellular distribution. Depletion of this tetraspanin changed $\beta 4$ integrin dissemination in SCC cells resulting in their impaired clustering and spreading. The molecular mechanism of this phenomenon is thought to be based on the CD151-mediated protein kinase $\mathrm{C} \alpha-\alpha 6 \beta 4$-integrin association. PKC is responsible for $\beta 4$ integrin phosphorylation (S1424) in talin and EGFR-dependent manner (induced by stimulation with phorbol ester and EGF, respectively). This activation triggers dissociation of integrin from intermediate filaments and disruption of hemidesmosomes, leading to a more invasive phenotype. ${ }^{11}$ Similar mechanisms involving $\beta 1$ integrin were also observed in erythroleukaemia and fibrosarcoma cells. ${ }^{51}$ In HB2 mammary epithelial cells, depletion of CD151 was shown to cause redistribution of $\alpha 3 \beta 1$ (but not of $\alpha 6$ ) integrin from cell-cell to cell-ECM contacts in 3D ECM growth resulting in partial restoration of cell polarity. Elevated expression of CD151 in DCIS was associated with proliferation and filling of the mammary gland lumen with cells, suggesting that CD151 may have an important role at the early stage of disease development. ${ }^{12}$

\section{Signalling}

CD151 is a master regulator of signalling mediated by laminin-binding integrins. It acts directly on signalling, that is, on downstream effectors crucial for motility of many types of cancer cells, for example, FAK, ${ }^{34,52} \mathrm{Src}$, Erk1/2, ${ }^{53} \mathrm{p} 38,{ }^{26}$ Racl and Ick ${ }^{34}$ in BCa cell lines; FAK, Src, p130Cas and paxillin in human lung adenocarcinoma cell line A549; ${ }^{43} \mathrm{FAK}$, Src, p38 and JNK kinases in human melanoma cell lines. ${ }^{21,24}$

In the MDA-MB-231 mammary cancer cell line, the CD151- $\alpha 3 \beta 1$ integrin complex was shown to participate in invasive migration in $3 \mathrm{D}$ environment. This could be blocked by a specific inhibitor (LY294002) of PI3K or an actin filament stabilizing compound (jasplakinolide), suggesting that the tetraspanin-integrin complex may govern invasive migration through PI3K-dependent rearrangement of the actin cytoskeleton. ${ }^{7}$ In erythroleukaemic and fibrosarcoma cell lines, $\alpha 3 \beta 1$ showed the highest (of all integrins studied so far) activity of associated phosphatidylinositol-4-kinase (PI4Kconverts PtdIns into phosphatidylinositol-4-phosphate, an intermediate connected with phosphatidylinositol signalling pathways). This can be suppressed by CD151 immunodepletion. For example, in erythroleukaemia and fibrosarcoma cell lines, CD151 controlled $\alpha 3 \beta 1$ association with PI4K resulting in regulation of actin polymerization and cell migration. Both anti-CD151 and anti- $\alpha 3$ antibodies almost completely abolished chemotactic migration of cells, thus implicating the CD151-PtdIns-4-kinase- $\alpha 3 \beta 1$ integrin complex in cell motility. ${ }^{19}$

CD151 has also been shown to be involved in regulation of small GTPases function. Members of Ras and Rho (eg, RhoA,
Rac and Cdc42) family of GTPases regulate important cellular processes ranging from cytoskeletal remodelling and gene expression to cell proliferation and membrane trafficking. These proteins have been shown to have a central role in modulating integrin-dependent cell behaviors, including cell adhesion, migration and spreading. ${ }^{54}$ Aberrant Rho GTPases signalling has, therefore, a profound impact on various aspects of cancer pathophysiology. Importantly, CD151 overexpression was found to activate Rac, Cdc42, but not Rho in A431 epidermoid carcinoma cells. ${ }^{20}$ In human melanoma cell lines (C8161 and MelJuSo), CD151 was demonstrated to take part in the recruitment of Ras, Racl and Cdc 42 to the cell membrane region, rich in $\alpha 3 \beta 1 /$ $\alpha 6 \beta 1$ integrin-CD151-GTPase complexes. CD151 links these integrins to Ras, Rac1 and Cdc42 by promoting formation of the multimolecular membrane complexes during cell adhesion to laminin, which leads to upregulation of adhesiondependent small GTPase activation. GTPase activity was also demonstrated to be affected by homophilic CD151-mediated cell-to-cell adhesion. ${ }^{21}$ However, acting through this type of interaction was reported by only one group. The extracellular part of tetraspanins protrudes $\sim 5 \mathrm{~nm}$ from the cell membrane $^{55}$ which, together with much 'taller' $(\sim 20 \mathrm{~nm})$ associated integrins, makes direct homophilic binding between CD151's on neighboring cells physically rather unlikely. Fluorescence Resonance Energy Transfer (FRET) analysis showed that CD151 and $\alpha 3 \beta 1$ integrin associated at the front and retracting rear of polarized migrating breast carcinoma cells in 2D and 3D matrices. This complex promoted cellular invasion by regulating actin-based membrane protrusions and retraction. CD151 association with $\alpha 3 \beta 1$ occurs dynamically within discrete subcellular compartments and acts to establish local GTPase signalling to promote tumour cell invasion. ${ }^{56}$

Apart from its ability to mediate cell-ECM cross-talk, CD151 was also shown as an important mediator of the stability of tumour cell-cell interaction and collective cell migration of epidermal carcinoma cells via regulation of RhoA activity through its interaction with $\alpha 3 \beta 1$ integrin. Experimental studies carried out under conditions enabling maintenance of cell-cell contacts revealed that CD151 depletion led to enhanced collective cell migration of intact cell sheets. This was a result of excessive RhoA activation, destabilization of E-cadherin-based cell-cell contacts and loss of actin organization at the cell junctions as well as increased formation of stress fibers on the basal cell surface. ${ }^{22}$ On the other hand, silencing of CD151 in the same epidermal carcinoma cells was previously shown to impair single-cell migration velocity ${ }^{45}$ Therefore, it appears that $\mathrm{CD} 151-\alpha 3 \beta 1$ integrin complex has a role depending on the biological context; promoting, when engaged in single-cell migration or inhibitory, while in the collective cell movement. Authors supply an explanation for this paradox suggesting that factors that limit much faster single-cell velocity are not the velocitylimiting factors in slower collective migration. For collective 
cell migration, a major velocity-limiting factor might be simply the restraint imposed by being part of the collective (reduced in CD151-negative cells).

\section{Interactions with MMPs}

CD151 is also implicated in regulation of the expression and activity of MMPs (Figure 2). Functional association of CD151 with MMPs is undoubtedly one of the key mechanisms of its involvement in tumour progression. During invasion, ECM components are degraded by MMPs, often activated by the urokinase-type plasminogen activator (uPA). ${ }^{57}$ Proteases such as MMP-2, -9 and -14 (with integrins as principal adhesive components) are mostly concentrated near the focal contacts at invadopodias. ${ }^{58}$ The CD151- $\alpha 3 \beta 1$ integrin complex was found to regulate production of

\section{TUMOR MICROENVIRONMENT}

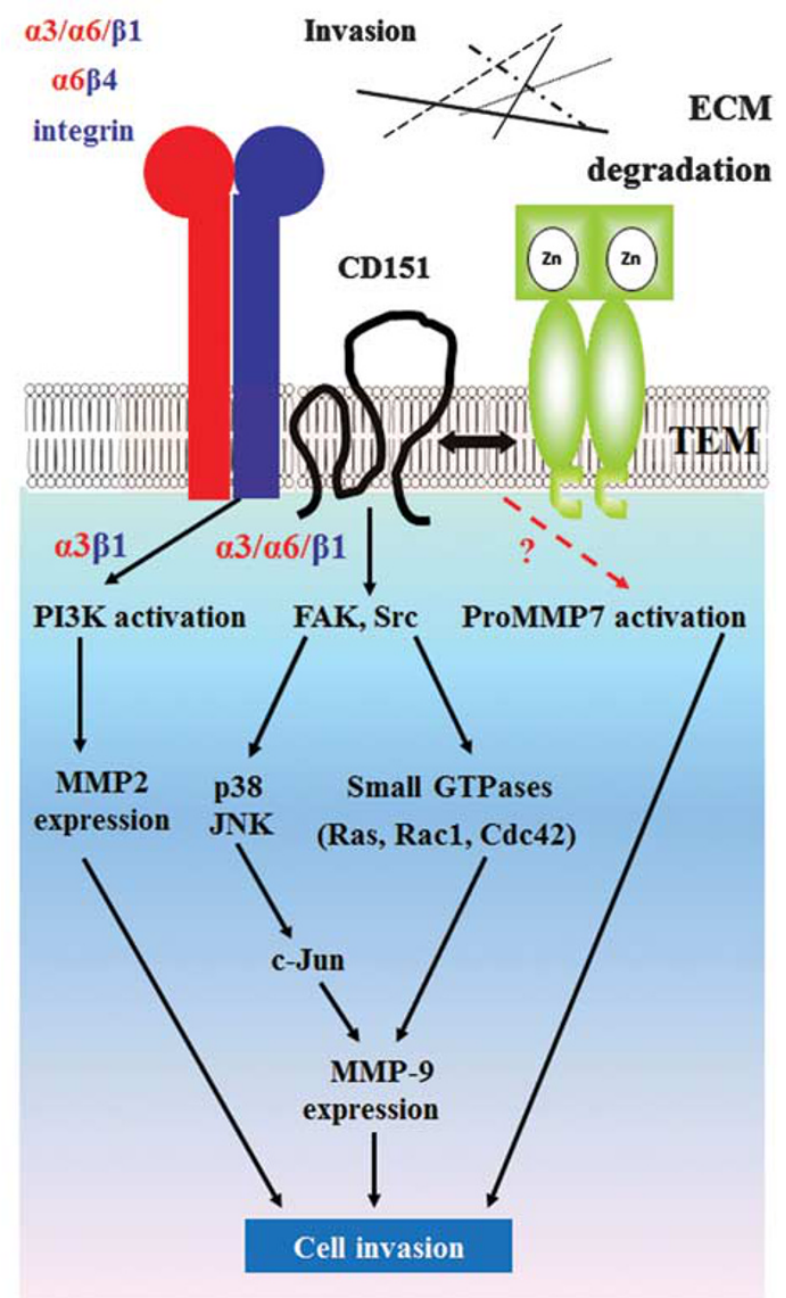

Figure 2 Complexity of CD151 action in cancer progression-interaction with metalloproteinases. CD151/laminin-binding integrin complexes regulate expression and activity of MMPs.
MMP-2 through the PI3K pathway in invasive cancer cells migrating within the 3D extracellular environment. ${ }^{7}$ Knockdown of CD151 in HCC cells resulted in reduced concentration of MMP-9. ${ }^{59}$ In rat pancreatic adenocarcinoma, CD151 was found to recruit and activate MMP-9 and MMP-13, creating a path for migrating cells. ${ }^{60}$

Homophilic CD151 interaction on the surface of neighboring human melanoma cells triggered integrin-dependent signalling leading to c-Jun binding to AP-1 sites in the MMP-9 gene promoter, followed by an increase in MMP-9 expression, finally resulting in cellular invasion..$^{24}$ Moreover, CD151 was recruited to the cell membrane and stimulated activation of small GTPases, which resulted in induction of MMP-9 expression and cell motility. ${ }^{21} \mathrm{CD} 151$ was identified as a proMMP-7 binding protein in a yeast two-hybrid screen. ${ }^{61}$ Formation of the complex was demonstrated by co-immunoprecipitation and co-localization of proMMP-7 with CD151 at the leading edge of the lamellipodia of human epidermal and rectal carcinoma cell lines. ProMMP-7 was captured and activated on the cell membrane of cultured cells through interaction with CD151. MMP-7 activity was also confirmed around the human lung adenocarcinoma cell nests, the sites of CD151 and MMP-7 colocalization. ${ }^{61,62}$ However, reported by Shiomi et al a remarkably strong interaction of CD151 with pro-MMP-7, observed even in stringent conditions, has not been so far confirmed by other groups. Moreover, presented recovery of interaction was supposed to involve the large extracellular loop (EC2) of CD151. The fact that conformation of EC2 is known to be highly dependent on multiple disulfide bonds, which would not be present in the yeast during the screen, casts serious doubts on the scientific value of the reported observation.

CD151-mediated formation of ternary complex (identified by immunoprecipitation and FRET analyses) between CD151- $\alpha 3 \beta 1$ integrin and membrane-associated protease MT1-MMP (MMP-14) has been shown in endothelial cells. Studies on HUVEC and mouse lung endothelial cells (MLECs) suggest that CD151 functions as a linker between MT1-MMP and $\alpha 3 \beta 1$ integrin directing MT1-MMP to endothelial lateral junctions regulating its colagenolytic activity. ${ }^{63}$ Thus, it is conceivable that CD151, acting as a regulator of endothelial homeostasis, may indirectly contribute to tumor growth and metastasis.

\section{Role in Growth Factor-Mediated Interplay between Tumour and Its Microenvironment}

It is widely accepted that development of carcinoma is strongly influenced by the tumour microenvironment. Its cellular component consists of fibroblasts, blood vasculature and inflammatory cells and is a rich source of growth factors and chemokines. Increasing evidence strongly points to the involvement of CD151 in the regulation of growth factor receptor function and their cross-talk with integrins on the cell surface (Figure 3). CD151/integrin $\alpha 3 / \alpha 6$ complex formed a structural and functional association with c-Met 


\section{TUMOR MICROENVIRONMENT}

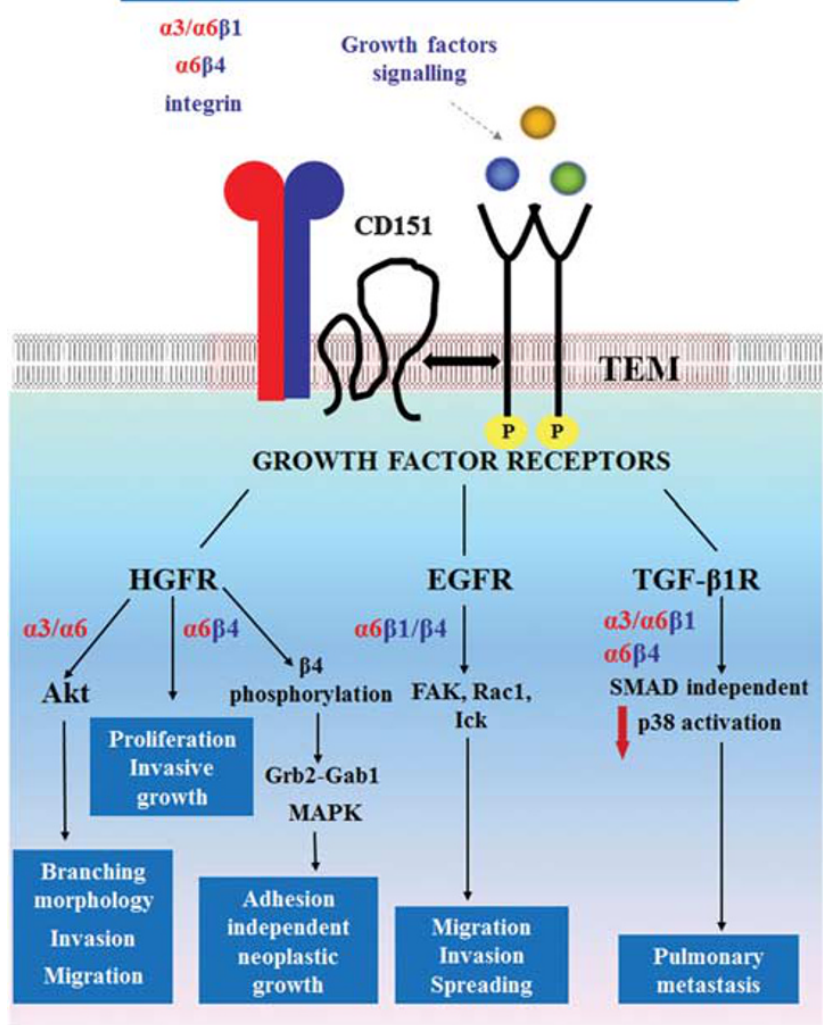

Figure 3 Complexity of CD151 action in cancer progression-role in growth factor receptors signalling. CD151/laminin-binding integrin complexes are involved in communication with tumour microenvironment mediating growth factor receptors activities.

(hepatocyte growth factor receptor) and regulated c-Met/ HGF-driven oncogenic activity of salivary gland cancer cells. ${ }^{33}$ In $\mathrm{BCa}$ cells grown on matrigel, these heteromeric assemblies promoted branching morphogenesis by regulating HGF-dependent signalling (reduced Akt activation in CD151-negative cells). ${ }^{64} \mathrm{CD} 151$ is also implicated in the formation of signalling complexes between cMet and $\beta 4$ integrin, which are known to enhance proliferation and invasive growth of neoplastic cells upon interaction with HGF. In vivo, CD151 was shown to mediate HGF-driven tumour progression, the mechanism involving $\beta 4$ integrin phosphorylation by c-Met followed by Grb2-Gab1 association and MAPK stimulation. ${ }^{65}$ In breast carcinoma, CD151 was also shown to mediate communication of cancer cells with the tumour microenvironment by linking integrins with the receptor for endothelium-derived growth factor, thus supporting in vivo tumourigenesis. ${ }^{53} \mathrm{~A}$ regulatory role of CD151 in tumour-stroma interplay was demonstrated in transforming growth factor- $\beta$ (TGF- $\beta$ )-mediated cross-talk between $\mathrm{BCa}$ metastatic cells and lung parenchyma. CD151 was shown to modulate responsiveness of cancer cells to TGF- $\beta$ produced by pneumocytes, implicating the tetraspanin in cell homing to the metastatic niche and, consequently, enhancing metastatic burden and spread of the disease. ${ }^{26}$ In BCa cells (MDA-MB-231), CD151 ablation was proven to disrupt EGFR- $\alpha 6$ integrin interaction subsequently affecting cell migration, invasion and spreading in response to $\mathrm{EGF}^{34}$

Existing studies describing CD151 involvement in communication between tumour and its microenvironment have primarily focussed on an impact of its presence on the membrane of cancer cells on cellular response to the stimuli generated by the stroma. As CD151 is ubiquitously expressed, its contribution to the reverse feedback, that is, the interactions between CD151-expressing stromal cells and the tumour, or even more systemic response involving the immune system, are highly probable. These aspects of CD151 biology are still waiting to be addressed and fully revealed. A role of endothelium-expressed CD151 in cancer development is discussed below.

\section{Involvement in Angiogenesis}

Formation of blood vessels in support of tumour growth is a fundamental process leading to cancer progression. Neoangiogenesis and subsequent maintenance of tumour vasculature are thought to be supported by reciprocal interactions between endothelial and cancer cells. This interplay is also crucial for intravasation and extravasation, prerequisite events in metastasis. ${ }^{66} \mathrm{CD} 151$ is commonly considered as an important modulator of adhesive interactions in both endothelial and cancer cells. CD151-null mice showed no vascular abnormalities during normal development but deletion of CD151 resulted in both in vitro and in vivo impairment of angiogenesis, that is, disruption of endothelial cell migration, spreading and invasion. CD151-depleted endothelial cells demonstrated significant alterations in adhesion-dependent activation of $\mathrm{PKB} / \mathrm{c}-\mathrm{Akt}$, e-NOS, Rac and Cdc42. ${ }^{13}$ As demonstrated by Liu et al, ${ }^{67}$ CD151mediated angiogenesis and activation of signalling pathways rely on formation of CD151/integrin complexes. A recent study by Zhang et al ${ }^{15}$ implicated CD151 in stabilization of endothelial capillary-like structures formed in vitro, maintaining the balance between adhesion of endothelial cells and their cytoskeletal flexibility as well as integration of cell-ECM and endothelial cell-cell adhesive interactions in vivo. These results are consistent with a postulated role of CD151 as a promoter of tumourigenesis in BCa cells, which mediates communication between the tumour and endothelial cells. ${ }^{53}$ Furthermore, CD151-null mice injected with melanoma cells showed a significantly diminished metastatic burden due to the altered interaction between tumour and endothelium. ${ }^{14}$ Thus, the regulatory role of the tetraspanin CD151 in integrin $(\alpha 3 \beta 1, \alpha 6 \beta 1$ and $\alpha 6 \beta 4)$ ligandbinding and signalling functions in both endothelial and cancer cells implicates the protein as one of the key players in pathologic angiogenesis, a process crucial for cancer progression and spread. 


\section{CLINICAL ASPECTS OF CD151 EXPRESSION}

Clinically, high levels of CD151 are correlated with poor prognosis in a variety of tumours including epithelial malignancies such as carcinomas of the lung, ${ }^{68}$ breast (invasive ductal carcinoma), $, 34,53,69$ colon, ${ }^{70}$ pancreas, ${ }^{71}$ kidney, ${ }^{10}$ prostate $(\mathrm{PCa}),{ }^{72}$ liver $^{73,74}$ and oesophagus (SCC), ${ }^{75}$ as well as glioblastoma. ${ }^{76}$

As in most cancers an elevated level of CD151 expression is associated positively with a high tumour grade (Table 1), the assumption that CD151 is involved in cancer progression is well grounded. In particular, in prostate cancer, expression of this tetraspanin was found to be markedly higher in poorly differentiated PCa and was demonstrated to have better prognostic value than traditional Gleason grading for prediction of clinical outcome of the patients. ${ }^{72}$

Clinical implications of CD151 expression have widely been studied in $\mathrm{BCa}$. Three comprehensive analyses emphasized CD151's contribution to cancer progression. All studies led to the conclusion that elevated expression of CD151 significantly correlated with tumour size, stage, nodal status, and ER- (oestrogen receptor), PR- (progesterone receptor) and HER2-negative phenotype, with minor differences, which resulted most likely from inconsistencies in either methodology or criteria for sample selection. Multivariate analyses showed that in invasive ductal BCa (IDC), CD151 overexpression was independently correlated with poor survival. ${ }^{34,53} \mathrm{~A}$ more recent study of a cohort categorized into five BCa subtypes (according to ER, PR, HER2, EGFR and CK5/6 expression) demonstrated that CD151 overexpression retained its adverse impact on survival in the luminal $\mathrm{A}$ $(\mathrm{ER}+/ \mathrm{PR}+/ \mathrm{HER} 2-)$ and quintuple-negative $\mathrm{BCa}$ subtypes (ER - /PR - /HER2 - /EGFR - /CK5/6 - ), a subgroup of triple-negative $\mathrm{BCa}^{69}$ In addition, increased expression of CD151 was observed in premalignant form of BCa (DCIS), ${ }^{12}$ suggesting that CD151, combined with interacting molecules may also have a crucial role at the early, proliferative stages of the disease.

An immunohistochemical study of endometrial cancer revealed that the level and prognostic value of CD151 expression differed between tumour phenotypic subtypes. Expression was significantly higher in uterine papillary serous (UPSC) and clear cell carcinoma (CC) than in high-grade endometrioid carcinoma (G3 EEC), sarcoma or carcinosarcoma. Univariate and multivariate analyses highlighted CD151 as an independent prognostic marker in UPSC, $\mathrm{CC}$ subtypes and triple-negative tumours (ER $-/ \mathrm{PR}-/$ HER2 - ). However, high level of CD151 expression positively correlated with improved survival, suggesting that in endometrial carcinoma, unlike in other epithelial cancers, CD151 might have a suppressive role in transition from normal to malignant phenotype. It was demonstrated that in endometrial carcinoma cells, expression of CD151 was strongly linked to that of E-cadherin but not to $\alpha 3 \beta 1$ or $\alpha 6 \beta 1$ integrins. The mechanism underlying the unexpected expression of CD151, however, was not further investigated. ${ }^{77}$
CD151 was found to be of no prognostic value in colorectal and urothelial bladder cancers. Moreover, its expression in malignant cells was downregulated when compared with normal adjacent tissue. ${ }^{9,78}$ No association with disease progression was demonstrated in either oral SCC or epithelial ovarian tumours by immunochemical and transcriptional analysis, respectively. ${ }^{79,80}$

The ability of CD151 to enhance primary tumour growth and promote cancer cell invasion and migration in vitro has aroused an interest in its potential contribution to metastasis. Indeed, overexpression of CD151 at the primary site was found to correspond with the development of metastases, mostly in lymph nodes. ${ }^{53,71,73-75}$ Formation of secondary tumours in distant organs such as lungs and bone was also noted in clear cell renal carcinoma (RCC). ${ }^{10}$

CD151 is implicated as a potential diagnostic marker in osteosarcoma and prostate cancer. ${ }^{81,82}$ Recent proteomic analysis of microvesicles released into the extracellular environment by the metastatic prostate cancer cell line PC3 revealed an enrichment in CD151. ${ }^{82}$ Future clinical studies of biological fluids are needed to validate whether CD151 fulfils its potential as a prognostic biomarker.

CD151's role as a promoter of cancer progression implicates the protein as a putative target for antibody-based immunotherapy. The potential efficacy of anti-CD151 monoclonal antibodies (mAbs) have been proposed in numerous studies reviewed by Haeuw et al. ${ }^{83}$ For example, an antagonistic effect may be achieved by blocking interactions with one of CD151's key molecular partners, using the 8C3 $\mathrm{mAb}$, which has been shown to dissociate CD151 from $\alpha 3 \beta 1$ integrin and reduce $\alpha 3 \beta 1$-mediated adhesion to laminin by $\sim 50 \% .{ }^{40}$ Anti-CD151 mAbs could also act indirectly by disrupting interactions between growth factor receptors and integrins. Alternatively, agonistic effects such as activation/ clustering of CD151's partners could be induced. For example, an increase in integrin-related adhesion forces was shown to prevent tumour cell motility. In support of this, supplementation with mAb $1 \mathrm{~A} 5$ resulted in a stronger cellmatrix adhesion, most likely due to increased integrin avidity and adhesion. Another mode of antimetastatic action to be considered involves induction/strengthening of cell-cell contacts, for example, using the mAb 11B1.G4. ${ }^{25,84}$

Numerous antibodies have been tested in vivo and shown to inhibit metastasis. These include $50-6,{ }^{6}$ SFA1.2B $4^{8}$ and $1 \mathrm{~A} 5 .^{25}$ Treatment with the mAb 50-6 reduced lung metastases formed by Hep3 epidermoid carcinoma cells in chicken embryos to $57 \%$, but had no effect on the size of the primary tumours. Blockade of metastasis formation was more effective with antibodies injected within $2-6 \mathrm{~h}$ of inoculation of the cells. This suggests that CD151 may act in the early events of the metastatic cascade such as cell adhesion to the vessel wall, extravasation and/or tumour cell migration to the sites of secondary growth. ${ }^{6}$ Inhibition of cancer cell motility in vivo, in response to $1 \mathrm{~A} 5 \mathrm{mAb}$ treatment, was demonstrated by real-time imaging. ${ }^{25}$ It was shown that the 
Table 1 CD151 expression and its prognostic significance in human cancers

Correlation with

\begin{tabular}{|c|c|c|c|c|c|c|c|c|c|c|}
\hline & & Expressi & of CD151 & Relati & $\mathrm{n}$ with tur & or characteristics & & gnosis & & \\
\hline Cancer & & Upregulation & Downregulation & Grade & Size & Metastasis & Positive & Negative & Other markers & Reference \\
\hline Breast & & $38 / 124$ & & Positive & & & & & ER negative & 34 \\
\hline Subtypes & $\mathrm{ER}+/ \mathrm{HER} 2-$ & $9 / 38$ & & & & & & & & \\
\hline & ER + /HER2 + & $7 / 38$ & & & & & & & & \\
\hline & ER - /HER2 + & $6 / 38$ & & & & & & & & \\
\hline & ER - /HER2 - & $19 / 38$ & & & & & & & & \\
\hline Breast (ductal & & $17 / 56$ & & & & Positive & & OS & & 53 \\
\hline Breast & & $127 / 886$ & & & Positive & Positive & & OS & HER2 positive & 69 \\
\hline & & & & & & & & & ER/PR negative & \\
\hline Subtypes & Luminal A & $44 / 451$ & & & & & & OS & & \\
\hline & Luminal B & $19 / 113$ & & & & & & OS & & \\
\hline & HER2 & $29 / 106$ & & & & & & & & \\
\hline & TNBC & $35 / 216$ & & & & & & & & \\
\hline & BLBC & $22 / 135$ & & & & & & & & \\
\hline & QNBC & $12 / 81$ & & & & & & & & \\
\hline Prostate & & $7 / 30$ & & Positive & & & & OS & & 72 \\
\hline Hepatocellula & & $311 / 520$ & & Positive & Positive & Positive & & $O S$ and $C R$ & c-Met & 74 \\
\hline Intrahepatic & ingiocarcinoma & $75 / 140$ & & Positive & Positive & Positive & & $O S$ and $C R$ & & 73 \\
\hline Non-small ce & & $86 / 145$ & & & & & & OS & & 68 \\
\hline Pancreatic du & & $35 / 71$ & & & & Positive & Positive & OS & $\begin{array}{l}\text { c-Met, integrin } \alpha 3 / \alpha 6 \\
\text { positive }\end{array}$ & 71 \\
\hline Renal cell car & & $232 / 489$ & & Positive & Positive & Positive & & PFS and DSS & & 10 \\
\hline & & & & & & Lymph nodes, lung, & & & & \\
\hline & & & & & & bone positive & & & & \\
\hline
\end{tabular}

$\begin{array}{clc}\text { Endometrial } & & 72 / 131 \\ \text { Group I } & \text { G3 EEC } & 25 / 68 \\ \text { Group II } & \text { USPC } & 30 / 31 \\ & \text { CC } & 7 / 7 \\ \text { Group III } & \text { Sarcoma } & 4 / 13 \\ & \text { MMMT } & 1 / 9 \\ & \text { Mixed mesodermal } & 0 / 3 \\ & \text { tumours } & \end{array}$




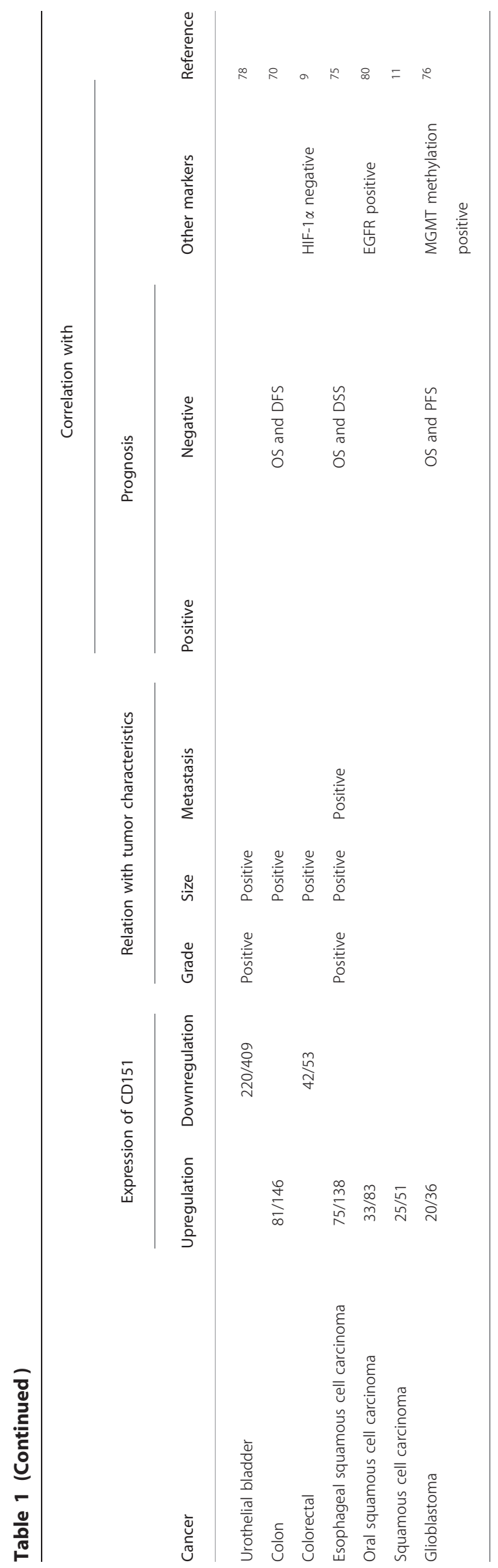

treatment caused an inability to detach at the rear of the cell and to form an invasive edge in the primary tumour. Moreover, application of anti-CD151 mAbs prevented intravasation (but not extravasation) and inhibited spontaneous metastasis of Hep3 (epidermoid carcinoma) and HT1080 (fibrosarcoma) cells in chicken embryos and SCID mice (>80\%). MAb SFA1.2B4 was shown to decrease to $64-75 \%$ pulmonary metastases formed by RPMI4788 and HT1080 cells overexpressing CD151 in BALB/c nu/nu mice. ${ }^{8}$ Although targeting of CD151 with mAbs holds promise for cancer immunotherapy, further studies are required to overcome obstacles, especially those caused by ubiquitous expression of CD151, and hence, an inability to limit efficacy of the target compound to the affected organ.

Recent evidence from in vitro studies point to another aspect of CD151 function that might bear significant clinical implications. Using a panel of ERRB2-positive BCa cell lines (MDA-MB-453, SKBR3, BT474 and ZR75), it has been shown that CD151/laminin-binding integrins complexes mediated adhesion to laminin- 5 and provided resistance to anti-ERBB2 agents. ${ }^{85}$ Additionally, CD151 and interacting integrins cooperated with ERBB2 receptor in regulating multiple signalling pathways (ie, ERK, FAK and caspase-3 activation), thereby driving progression of mammary tumour. ${ }^{86}$ These data strongly suggest that CD151 might be involved in the regulation of ERBB2 function and hence has a role in the development of resistance to anti-ERBB2 (eg, herceptin and lapatinib) agents.

Although at first glance CD151 appears to be merely a structural protein of the cell membrane with no binding ligand and/or enzymatic activity (intracellular effectors directly interacting with CD151 have not been identified yet), numerous studies demonstrate its contribution to the cancer progression. Multiple lines of evidence, that is, the ability of CD151 to modulate tumour cell activities mediated by laminin-binding integrins, MMPs and growth factor receptors clearly show a multi-faceted nature of involvement of this tetraspanin in carcinogenesis. Taken together, these data strongly implicate CD151 as a potential diagnostic, prognostic marker as well as a target for cancer therapy.

\section{ACKNOWLEDGMENTS}

We thank Dr Stephen F Murphy, Dr Sven H Petersen and Dr Anna J Zaczek for critically reading the manuscript. This work was supported by Foundation for Polish Science (HOMING PLUS/2010-2/12) and National Science Centre (UMO-2011/01/B/NZ4/04910) grants.

\section{AUTHOR CONTRIBUTIONS}

$\mathrm{RS}$ and HMR prepared the manuscript and wrote the final version. AG, LT and RK contributed with specific section. All authors read and approved the final manuscript.

\section{DISCLOSURE/CONFLICT OF INTEREST}

The authors declare no conflict of interest.

1. Sincock PM, Mayrhofer G, Ashman LK. Localization of the transmembrane 4 superfamily (TM4SF) member PETA-3 (CD151) in normal human tissues: comparison with CD9, CD63, and alpha5beta1 integrin. J Histochem Cytochem 1997;45:515-525. 
2. Zöller M. Tetraspanins: push and pull in suppressing and promoting metastasis. Nat Rev Cancer 2009;9:40-55.

3. Berditchevski F. Complexes of tetraspanins with integrins: more than meets the eye. J Cell Sci 2001;114(Pt 23):4143-4151.

4. Lazo PA. Functional implications of tetraspanin proteins in cancer biology. Cancer Sci 2007;98:1666-1677.

5. Romanska HM, Berditchevski F. Tetraspanins in human epithelial malignancies. J Pathol 2011;223:4-14.

6. Testa JE, Brooks PC, Lin JM, et al. Eukaryotic expression cloning with an antimetastatic monoclonal antibody identifies a tetraspanin (PETA-3/ CD151) as an effector of human tumor cell migration and metastasis. Cancer Res 1999;59:3812-3820.

7. Sugiura T, Berditchevski F. Function of alpha3beta1-tetraspanin protein complexes in tumor cell invasion. Evidence for the role of the complexes in production of matrix metalloproteinase 2 (MMP-2). J Cell Biol 1999;146:1375-1389.

8. Kohno M, Hasegawa $H$, Miyake $M$, et al. CD151 enhances cell motility and metastasis of cancer cells in the presence of focal adhesion kinase. Int J Cancer 2002;97:336-343.

9. Chien CW, Lin SC, Lai YY, et al. Regulation of CD151 by hypoxia controls cell adhesion and metastasis in colorectal cancer. Clin Cancer Res 2008;14:8043-8051.

10. Yoo SH, Lee K, Chae JY, et al. CD151 expression can predict cancer progression in clear cell renal cell carcinoma. Histopathology 2011;58:191-197.

11. Li Q, Yang $\mathrm{XH}, \mathrm{Xu} F$, et al. Tetraspanin CD151 plays a key role in skin squamous cell carcinoma. Oncogene 2013;32:1772-1783.

12. Novitskaya V, Romanska H, Dawoud M, et al. Tetraspanin CD151 regulates growth of mammary epithelial cells in three-dimensiona extracellular matrix: implication for mammary ductal carcinoma in situ. Cancer Res 2010;70:4698-4708.

13. Takeda $Y$, Kazarov AR, Butterfield CE, et al. Deletion of tetraspanin Cd151 results in decreased pathologic angiogenesis in vivo and in vitro. Blood 2007;109:1524-1532.

14. Takeda Y, Li Q, Kazarov AR, et al. Diminished metastasis in tetraspanin CD151-knockout mice. Blood 2011;118:464-472.

15. Zhang F, Michaelson JE, Moshiach S, et al. Tetraspanin CD151 maintains vascular stability by balancing the forces of cell adhesion and cytoskeletal tension. Blood 2011;118:4274-4284.

16. Bari R, Guo Q, Xia B, et al. Tetraspanins regulate the protrusive activities of cell membrane. Biochem Biophys Res Commun 2011;415:619-626.

17. Yánez-Mó $M$, Mittelbrunn M, Sánchez-Madrid F. Tetraspanins and intercellular interactions. Microcirculation 2001;8:153-168.

18. Peñas PF, García-Díez A, Sánchez-Madrid F, et al. Tetraspanins are localized at motility-related structures and involved in normal human keratinocyte wound healing migration. J Invest Dermatol 2000;114: 1126-1135.

19. Yauch RL, Berditchevski F, Harler MB, et al. Highly stoichiometric, stable, and specific association of integrin alpha3beta1 with CD151 provides a major link to phosphatidylinositol 4-kinase, and may regulate cell migration. Mol Biol Cell 1998;9:2751-2765.

20. Shigeta M, Sanzen N, Ozawa M, et al. CD151 regulates epithelial cellcell adhesion through PKC- and Cdc42-dependent actin cytoskeletal reorganization. J Cell Biol 2003;163:165-176.

21. Hong IK, Jeoung $\mathrm{DI}, \mathrm{Ha} \mathrm{KS}$, et al. Tetraspanin CD151 stimulates adhesion-dependent activation of Ras, Rac, and Cdc42 by facilitating molecular association between $\beta 1$ integrins and small GTPases. J Biol Chem 2012;287:32027-32039.

22. Johnson JL, Winterwood N, DeMali KA, et al. Tetraspanin CD151 regulates $\mathrm{RhoA}$ activation and the dynamic stability of carcinoma cellcell contacts. J Cell Sci 2009;122(Pt 13):2263-2273.

23. Ke AW, Shi GM, Zhou J, et al. CD151 amplifies signaling by integrin $\alpha 6 \beta 1$ to PI3K and induces the epithelial-mesenchymal transition in HCC cells. Gastroenterology 2011;140:1629-1641.e1615.

24. Hong IK, Jin YJ, Byun HJ, et al. Homophilic interactions of Tetraspanin CD151 up-regulate motility and matrix metalloproteinase-9 expression of human melanoma cells through adhesion-dependent c-Jun activation signaling pathways. J Biol Chem 2006;281: 24279-24292.

25. Zijlstra A, Lewis J, Degryse B, et al. The inhibition of tumor cell intravasation and subsequent metastasis via regulation of in vivo tumor cell motility by the tetraspanin CD151. Cancer Cell 2008;13: $221-234$.
26. Sadej $R$, Romanska H, Kavanagh D, et al. Tetraspanin CD151 regulates transforming growth factor beta signaling: implication in tumor metastasis. Cancer Res 2010;70:6059-6070.

27. Copeland BT, Bowman MJ, Ashman LK. Genetic ablation of the tetraspanin CD151 reduces spontaneous metastatic spread of prostate cancer in the TRAMP model. Mol Cancer Res 2013;11:95-105.

28. Wang $H X, L i ~ Q$, Sharma C, et al. Tetraspanin protein contributions to cancer. Biochem Soc Trans 2011;39:547-552.

29. Hasegawa $\mathrm{H}$, Watanabe $\mathrm{H}$, Nomura $\mathrm{T}$, et al. Molecular cloning and expression of mouse homologue of SFA-1/PETA-3 (CD151), a member of the transmembrane 4 superfamily. Biochim Biophys Acta 1997; 1353:125-130.

30. Fitter S, Seldin MF, Ashman LK. Characterisation of the mouse homologue of CD151 (PETA-3/SFA-1); genomic structure, chromosomal localisation and identification of 2 novel splice forms. Biochim Biophys Acta 1998;1398:75-85.

31. Fitter S, Tetaz TJ, Berndt MC, et al. Molecular cloning of CDNA encoding a novel platelet-endothelial cell tetra-span antigen, PETA-3. Blood 1995;86:1348-1355.

32. Kazarov AR, Yang X, Stipp CS, et al. An extracellular site on tetraspanin CD151 determines alpha 3 and alpha 6 integrin-dependent cellular morphology. J Cell Biol 2002;158:1299-1309.

33. Klosek SK, Nakashiro K, Hara S, et al. CD151 forms a functional complex with c-Met in human salivary gland cancer cells. Biochem Biophys Res Commun 2005;336:408-416.

34. Yang XH, Richardson AL, Torres-Arzayus MI, et al. CD151 accelerates breast cancer by regulating alpha 6 integrin function, signaling, and molecular organization. Cancer Res 2008;68:3204-3213.

35. Fujita Y, Shiomi T, Yanagimoto $S$, et al. Tetraspanin CD151 is expressed in osteoarthritic cartilage and is involved in pericellular activation of pro-matrix metalloproteinase 7 in osteoarthritic chondrocytes. Arthritis Rheum 2006;54:3233-3243.

36. Serru V, Le Naour F, Billard M, et al. Selective tetraspan-integrin complexes (CD81/alpha4beta1, CD151/alpha3beta1, CD151/alpha6beta1) under conditions disrupting tetraspan interactions. Biochem J 1999;340(Pt 1): 103-111.

37. Sterk LM, Geuijen CA, Oomen LC, et al. The tetraspan molecule CD151, a novel constituent of hemidesmosomes, associates with the integrin alpha6beta4 and may regulate the spatial organization of hemidesmosomes. J Cell Biol 2000;149:969-982.

38. Sterk LM, Geuijen CA, van den Berg JG, et al. Association of the tetraspanin CD151 with the laminin-binding integrins alpha3beta1, alpha6beta1, alpha6beta4 and alpha7beta1 in cells in culture and in vivo. J Cell Sci 2002;115(Pt 6):1161-1173.

39. Zevian S, Winterwood NE, Stipp CS. Structure-function analysis of tetraspanin CD151 reveals distinct requirements for tumor cell behaviors mediated by $\alpha 3 \beta 1$ versus $\alpha 6 \beta 4$ integrin. J Biol Chem 2011;286:7496-7506.

40. Nishiuchi R, Sanzen N, Nada S, et al. Potentiation of the ligand-binding activity of integrin alpha3beta1 via association with tetraspanin CD151. Proc Natl Acad Sci USA 2005;102:1939-1944.

41. Yang $\mathrm{XH}$, Mirchev $\mathrm{R}$, Deng $\mathrm{X}$, et al. CD151 restricts the $\alpha 6$ integrin diffusion mode. J Cell Sci 2012;125(Pt 6):1478-1487.

42. Lau LM, Wee JL, Wright MD, et al. The tetraspanin superfamily member CD151 regulates outside-in integrin alphallbbeta3 signaling and platelet function. Blood 2004;104:2368-2375.

43. Yamada M, Sumida Y, Fujibayashi $A$, et al. The tetraspanin CD151 regulates cell morphology and intracellular signaling on laminin-511. FEBS J 2008;275:3335-3351.

44. Sincock PM, Fitter S, Parton RG, et al. PETA-3/CD151, a member of the transmembrane 4 superfamily, is localised to the plasma membrane and endocytic system of endothelial cells, associates with multiple integrins and modulates cell function. J Cell Sci 1999;112(Pt 6): 833-844.

45. Winterwood NE, Varzavand A, Meland MN, et al. A critical role for tetraspanin CD151 in alpha3beta1 and alpha6beta4 integrin-dependent tumor cell functions on laminin-5. Mol Biol Cell 2006;17:2707-2721.

46. Liu L, He B, Liu WM, et al. Tetraspanin CD151 promotes cell migration by regulating integrin trafficking. J Biol Chem 2007;282:31631-31642.

47. Gesierich S, Paret C, Hildebrand D, et al. Colocalization of the tetraspanins, CO-029 and CD151, with integrins in human pancreatic adenocarcinoma: impact on cell motility. Clin Cancer Res 2005;11: 2840-2852. 
48. Baldwin G, Novitskaya V, Sadej R, et al. Tetraspanin CD151 regulates glycosylation of (alpha)3(beta)1 integrin. J Biol Chem 2008;283: 35445-35454.

49. Yang X, Claas C, Kraeft SK, et al. Palmitoylation of tetraspanin proteins: modulation of CD151 lateral interactions, subcellular distribution, and integrin-dependent cell morphology. Mol Biol Cell 2002;13:767-781.

50. Berditchevski $F$, Odintsova $E$, Sawada $S$, et al. Expression of the palmitoylation-deficient CD151 weakens the association of alpha 3 beta 1 integrin with the tetraspanin-enriched microdomains and affects integrin-dependent signaling. J Biol Chem 2002;277: 36991-37000

51. Zhang XA, Bontrager AL, Hemler ME. Transmembrane-4 superfamily proteins associate with activated protein kinase C (PKC) and link PKC to specific beta(1) integrins. J Biol Chem 2001;276:25005-25013.

52. Berditchevski F, Odintsova E. Characterization of integrin-tetraspanin adhesion complexes: role of tetraspanins in integrin signaling. J Cell Biol 1999;146:477-492.

53. Sadej R, Romanska H, Baldwin G, et al. CD151 regulates tumorigenesis by modulating the communication between tumor cells and endothelium. Mol Cancer Res 2009;7:787-798.

54. Wiesner S. Legate KR, Fässler R. Integrin-actin interactions. Cell Mol Life Sci 2005;62:1081-1099.

55. Min G, Wang H, Sun $T$ T, et al. Structural basis for tetraspanin functions as revealed by the cryo-EM structure of uroplakin complexes at 6-A resolution. J Cell Biol 2006;173:975-983.

56. Scales TM, Jayo A, Obara $B$, et al. $\alpha 3 \beta 1$ integrins regulate CD151 complex assembly and membrane dynamics in carcinoma cells within 3D environments. Oncogene 2013;32:3965-3979.

57. Clarke NW, Hart CA, Brown MD. Molecular mechanisms of metastasis in prostate cancer. Asian J Androl 2009;11:57-67.

58. Egeblad M, Werb Z. New functions for the matrix metalloproteinases in cancer progression. Nat Rev Cancer 2002;2:161-174.

59. Devbhandari RP, Shi GM, Ke AW, et al. Profiling of the tetraspanin CD151 web and conspiracy of CD151/integrin $\beta 1$ complex in the progression of hepatocellular carcinoma. PLoS ONE 2011;6:e24901.

60. Yue S, Mu W, Zöller M. Tspan8 and CD151 promote metastasis by distinct mechanisms. Eur J Cancer 2013:49:2934-2948.

61. Shiomi T, Inoki I, Kataoka F, et al. Pericellular activation of proMMP-7 (promatrilysin-1) through interaction with CD151. Lab Invest 2005; 85:1489-1506.

62. Hasegawa $M$, Furuya $M$, Kasuya $Y$, et al. CD151 dynamics in carcinomastroma interaction: integrin expression, adhesion strength and proteolytic activity. Lab Invest 2007;87:882-892.

63. Yanez-Mo M, Barreiro O, Gonzalo P, et al. MT1-MMP collagenolytic activity is regulated through association with tetraspanin CD151 in primary endothelial cells. Blood 2008;112:3217-3226.

64. Klosek SK, Nakashiro K, Hara S, et al. CD151 regulates HGF-stimulated morphogenesis of human breast cancer cells. Biochem Biophys Res Commun 2009:379:1097-1100.

65. Franco $M$, Muratori $C$, Corso $S$, et al. The tetraspanin CD151 is required for Met-dependent signaling and tumor cell growth. J Biol Chem 2010:285:38756-38764.

66. Al-Mehdi $A B$, Tozawa $K$, Fisher $A B$, et al. Intravascular origin of metastasis from the proliferation of endothelium-attached tumor cells: a new model for metastasis. Nat Med 2000;6:100-102.

67. Liu WF, Zuo HJ, Chai BL, et al. Role of tetraspanin CD151- $\alpha 3 / \alpha 6$ integrin complex: Implication in angiogenesis CD151-integrin complex in angiogenesis. Int J Biochem Cell Biol 2011;43:642-650.
68. Tokuhara $\mathrm{T}$, Hasegawa $\mathrm{H}$, Hattori $\mathrm{N}$, et al. Clinical significance of CD151 gene expression in non-small cell lung cancer. Clin Cancer Res 2001;7:4109-4114.

69. Kwon MJ, Park S, Choi JY, et al. Clinical significance of CD151 overexpression in subtypes of invasive breast cancer. Br J Cancer 2012;106: 923-930.

70. Hashida $\mathrm{H}$, Takabayashi A, Tokuhara $\mathrm{T}$, et al. Clinical significance of transmembrane 4 superfamily in colon cancer. $\mathrm{Br} J$ Cancer 2003;89:158-167.

71. Zhu GH, Huang C, Qiu ZJ, et al. Expression and prognostic significance of CD151, c-Met, and integrin alpha3/alpha6 in pancreatic ductal adenocarcinoma. Dig Dis Sci 2011;56:1090-1098.

72. Ang J, Lijovic M, Ashman LK, et al. CD151 protein expression predicts the clinical outcome of low-grade primary prostate cancer better than histologic grading: a new prognostic indicator? Cancer Epidemiol Biomarkers Prev 2004;13(11 Pt 1):1717-1721.

73. Huang XY, Ke AW, Shi GM, et al. Overexpression of CD151 as an adverse marker for intrahepatic cholangiocarcinoma patients. Cancer 2010;116:5440-5451.

74. Ke AW, Shi GM, Zhou J, et al. Role of overexpression of CD151 and/or c-Met in predicting prognosis of hepatocellular carcinoma. Hepatology 2009;49:491-503.

75. Suzuki S, Miyazaki T, Tanaka N, et al. Prognostic significance of CD151 expression in esophageal squamous cell carcinoma with aggressive cell proliferation and invasiveness. Ann Surg Oncol 2011;18:888-893.

76. Lee $\mathrm{D}$, Suh $\mathrm{YL}$, Park $\mathrm{Tl}$, et al. Prognostic significance of tetraspanin CD151 in newly diagnosed glioblastomas. J Surg Oncol 2013;107:646-652.

77. Voss MA, Gordon N, Maloney S, et al. Tetraspanin CD151 is a novel prognostic marker in poor outcome endometrial cancer. Br J Cancer 2011;104:1611-1618.

78. Minner $S$, De Silva $C$, Rink $M$, et al. Reduced CD151 expression is related to advanced tumour stage in urothelial bladder cancer. Pathology 2012;44:448-452.

79. Mosig RA, Lin L, Senturk E, et al. Application of RNA-Seq transcriptome analysis: CD151 is an Invasion/Migration target in all stages of epithelial ovarian cancer. J Ovarian Res 2012;5:4.

80. Romanska HM, Potemski P, Collins Sl, et al. Loss of CD151/Tspan24 from the complex with integrin $\alpha 3 \beta 1$ in invasive front of the tumour is a negative predictor of disease-free survival in oral squamous cell carcinoma. Oral Oncol 2013:49:224-229.

81. Zhang $Z$, Zhang $L$, Hua $Y$, et al. Comparative proteomic analysis of plasma membrane proteins between human osteosarcoma and normal osteoblastic cell lines. BMC Cancer 2010;10:206.

82. Sandvig K, Llorente A. Proteomic analysis of microvesicles released by the human prostate cancer cell line PC-3. Mol Cell Proteomics 2012:11:M111.012914.

83. Haeuw JF, Goetsch L, Bailly C, et al. Tetraspanin CD151 as a target for antibody-based cancer immunotherapy. Biochem Soc Trans 2011;39: 553-558.

84. Fitter $\mathrm{S}$, Sincock PM, Jolliffe $\mathrm{CN}$, et al. Transmembrane 4 superfamily protein CD151 (PETA-3) associates with beta 1 and alpha llb beta 3 integrins in haemopoietic cell lines and modulates cell-cell adhesion. Biochem J 1999;338(Pt 1):61-70.

85. Yang XH, Flores LM, Li Q, et al. Disruption of laminin-integrin-CD151focal adhesion kinase axis sensitizes breast cancer cells to ErbB2 antagonists. Cancer Res 2010;70:2256-2263.

86. Deng X, Li Q, Hoff J, et al. Integrin-associated CD151 drives ErbB2-evoked mammary tumor onset and metastasis. neoplasia 2012;14:678-689. 